\title{
Una taxonomía de materiales didácticos para la inclusión de alumnado con diversidad funcional auditiva
}

\section{A Taxonomy of Teaching Materials for the Inclusion of Students with Hearing Disability}

\author{
Carla Vázquez Formoso* \\ carlavazf@gmail.com \\ María Montserrat Castro Rodríguez* \\ maria.castror@udc.gal \\ Jesús Rodríguez Rodríguez**1 \\ jesus.rodriguez.rodriguez@usc.es \\ *Universidade da Coruña, España \\ **Universidade de Santiago de Compostela, España
}

\begin{abstract}
Resumen:
En este artículo se presenta una taxonomía, fruto de un trabajo de investigación, en relación con los materiales didácticos diseñados específicamente para las personas con discapacidad auditiva, pero también, se incorporan otros recursos y materiales que pueden favorecer la inclusión educativa de este colectivo en el aula ordinaria y en la sociedad. Presentamos en nuestra aportación una taxonomía que permita clasificar esta diversidad de materiales, concediéndole a la comunidad sorda, y al profesorado en general, la oportunidad de acceder a un sistema clasificatorio que les facilite la búsqueda de materiales y recursos.

En el trabajo se establecen, primeramente, los fundamentos teóricos del concepto de sordera y material didáctico y se describen sus características, clasificación y evolución a lo largo del tiempo. Después
\end{abstract}

\begin{abstract}
:
The present study presents a taxonomy developed during a research project in relation to didactic materials designed specifically for the hearing impaired. It also incorporates other resources that may foster the educational inclusion of this group in ordinary classrooms and society as a whole. The taxonomy makes it possible to classify a diversity of materials, thus providing the deaf community and teachers in general with a classification system that facilitates the search for materials and resources.

The paper first establishes the theoretical foundations of the concept of hearing impairment and didactic material and describes its characteristics, classification and evolution over time. After investigating diverse repositories and resource banks, the taxonomy resulting from the classification of different materials is presented. We
\end{abstract}

1 Dirección para correspondencia (correspondence address):

Jesús Rodríguez Rodríguez. Rúa Prof. Vicente Fraiz Andón, s/n. Campus Vida. 15782 Santiago de Compostela (España). 
Una taxonomía de materiales didácticos para la inclusión de alumnado con diversidad funcional auditiva

Carla Vázquez Formoso, María Montserrat Castro Rodríguez y Jesús Rodríguez Rodríguez

de realizar una investigación en los distintos repositorios y bancos de recursos, se expone la taxonomía a la que se llega como resultado de la clasificación de los distintos materiales; para concluir en una reflexión sobre los materiales mencionados y el proceso de elaboración de esta investigación.

Palabras clave:

Materiales didácticos; discapacidad auditiva; educación de sordos; taxonomía de los materiales; inclusión educativa. conclude with a reflection on the mentioned materials and how this research was carried out.

\section{Keywords:}

Teaching materials; hearing impairment; deaf education; materials taxonomy; educational inclusion.

\section{Résumé :}

Cet article présente une taxonomie, issue d'un travail de recherche, concernant les matériels didactiques spécifiquement conçus pour les personnes souffrant d'une déficience auditive, ainsi que d'autres ressources et matériels pouvant favoriser l'éducation inclusive de ces personnes dans les classes, et leur intégration dans la société. Dans le cadre de notre recherche, nous présentons une taxonomie permettant de classifier cette variété de matériels, et nous donnons à la communauté des sourds et des malentendants, et au corps enseignant en général, l'opportunité d'accéder à un système de classification qui facilite la recherche de matériels et de ressources.

Dans ce travail de recherche, nous établissons en premier lieu les fondements théoriques des concepts de surdité et de matériel didactique, et nous présentons une description de leurs caractéristiques, de leur classification et de leur évolution dans le temps. Après une étude des différents référentiels et banques de ressources, nous présentons la taxonomie à laquelle nous aboutissons, résultant de la classification des différents matériels, et nous concluons sur une réflexion concernant les matériels analysés et le processus d'élaboration de ce travail de recherche.

\section{Mots clés :}

Matériels didactiques; déficience auditive; éducation des sourds; taxonomie des matériels; éducation inclusive.

Fecha de recepción: 16-5-2017

Fecha de aceptación: 20-9-2017

\section{Introducción}

En el mercado actual podemos encontrar a disposición de la comunidad educativa un importante número de materiales específicos con los que trabajar con el alumnado con sordera, e igualmente, si revisamos congresos y experiencias relacionadas con el tema, podemos observar que el profesorado crea, intercambia y modifica materiales para utilizar con el alumno sordo. Conviene destacar que las nuevas tecnologías, se han constituido en un recurso muy favorecedor a la hora de ayudar en la producción de materiales didácticos para personas con discapacidad 
auditiva, ofreciendo como se indica por ejemplo en los trabajos de Domingo y Peñafiel (1998), Áfio, Carvalho y otros (2016), De Martino, Silva. y Bolognini, (2016), infinitas posibilidades de trabajo. Además, desde la perspectiva de la inclusión educativa, se debe promover la incorporación en el aula ordinaria de recursos y materiales que puedan ser utilizadas por todo el alumnado (Booth y Ainscow, 2002), independientemente de sus características auditivas. En las aulas ordinarias, el profesorado cada vez cuenta con más heterogeneidad en el aula. Evidentemente esta situación hace más complejo el proceso de diseño e implementación de propuestas educativas que den respuesta a las necesidades y potencialidades de todo el aula. Por este motivo, se necesita la introducción de estrategias y recursos lo suficientemente ricas, polivalentes y eficaces que faciliten la interacción a base del trabajo colaborativo o cooperativo entre estudiantes con perfiles muy diferenciados entre sí, ayudándoles a adquirir aquellas competencias y respetando las necesidades individuales y colectivas de los estudiantes. El hecho de que un material sea enriquecedor y sea accesible para un estudiante sordo, pero que también lo sea para sus compañeros y compañeras oyentes, no sólo facilita la interacción entre ellos, sino que a cada uno de ellos le facilitará la adquisición de competencias, de conocimientos compartidos y diferenciados de acuerdo con sus necesidades y potencialidades. Sin olvidar, la superación de estigmas sociales que aparecen en el mismo momento que conocemos o categorizamos los recursos en relación a una discapacidad o diversidad funcional y no en cuanto a las distintas capacidades u objetivos que se pueden alcanzar cada estudiante con dichos recursos. Esto es, en ocasiones, cuando un material se asocia directamente a un colectivo con diversidad funcional, sea de carácter sensorial, motórico, etc, no se tiene en consideración para usarlo con otras personas, con independencia de las características del material. Este desdén ocasiona que se haga un infrauso de dicho material, que podría ser interesante también para otras personas, pues los materiales, normalmente pueden tener un valor polivalente.

No obstante, uno de los problemas fundamentales con el que nos encontramos es la falta de una taxonomía que ayude a sistematizar las propuestas de materiales existentes con el propósito de servir de utilidad y dar respuesta a las necesidades del profesorado, alumnado, familias y otros miembros de la comunidad educativa. La propuesta de una taxonomía pretende aportar a la comunidad sorda una clara clasificación de los recursos que se encuentra a su alcance para utilizar cuando se con- 
sidere necesario. Igualmente, la propuesta pretende ayudar a identificar qué materiales pueden utilizarse específicamente con este alumnado, pero que también pueden ser útiles para el resto del alumnado del aula.

Conviene subrayar que si atendemos a la literatura existente sobre los materiales y su clasificación, es interesante resaltar el hecho de que la mayoría de las taxonomías existentes hasta ahora, están centradas fundamentalmente en los materiales didácticos en general (Véase por ejemplo la revisión de las clasificaciones de Area Moreira, 2009; Cabero, 2001; Rodríguez Rodríguez,2001; Rodríguez Rodríguez, y Rodríguez Regueira, 2016), y en algunos casos muy concretos en el ámbito de las necesidades educativas específicas. Por ejemplo, comprobamos la existencia de taxonomías que recogen materiales orientados a personas con movilidad reducida (Barragán, C., sf) o para la intervención en el ámbito de la comunicación (Rodríguez Cuello (2011) que ofrecen recursos organizados por temáticas, pero no incluyen materiales concebidos inicialmente para ser utilizados con fines didácticos, sino que suelen ser ayudas técnicas. En el caso concreto de la discapacidad auditiva, apenas encontramos algunos catálogos que presentan recursos técnicos que aunque no son pensados con carácter didáctico, si que pueden facilitar el acceso al conocimiento.

A través de la propuesta de una taxonomía para materiales didácticos elaborados para niños sordos nuestra principal pretensión es aportar a las personas sordas, profesorado, familiares, profesionales.... un esquema de clasificación sencillo de utilizar, a través del cual se facilite la busca de materiales de los que se pueden hacer uso cuando se precisen.

Con este propósito se desarrolló este trabajo que pretende atender a los siguientes objetivos específicos:

- Localizar, en el contexto español los materiales educativos diseñados para las personas sordas.

- Analizar y catalogar las propuestas de materiales recopilados de acuerdo con los ítems de la ficha de análisis elaborada.

- Presentar y difundir la taxonomía en la cual se clasifiquen los materiales didácticos.

\section{La discapacidad auditiva y los materiales didácticos}

Teniendo en cuenta los aspectos y características específicas y diversas de las personas con discapacidad auditiva, es necesario hacer hincapié 
en su escolarización y las modalidades educativas y de organización del currículum que se pueden dar. Autores como Domingo y Peñafiel (1998), sostienen que hay que tener en cuenta la etiología de la pérdida auditiva, el nivel intelectual del alumnado y las características del entorno, ya que las personas sordas forman un grupo muy heterogéneo. De esta manera, destacan dos modelos educativos, uno centrado en el déficit con el sujeto como propia causa de su problemática; y otro centrado en las competencias, asumiendo que cada persona es distinta y necesita un proceso de enseñanza-aprendizaje adaptado. En este sentido, Garrido, Martínez y Vázquez (1990), propusieron algunas estrategias para favorecer el proceso de cómo aplicar una metodología flexible y activa, implicando al alumnado con deficiencia auditiva en todas las actividades del aula presentando la información de manera visual y favoreciendo las interacciones con los compañeros. Esta situación en el acto educativo, significa la introducción en el aula de recursos que faciliten la adquisición del conocimiento a través del uso del resto de los sentidos (vista, gusto, tacto y olfato) o sistemas alternativos de comunicación (SAAC), para facilitar que el alumnado sordo pueda acceder al trabajo con el resto de sus compañeros en igualdad de oportunidades. No obstante, consideramos oportuno hacer hincapié en que el uso de estos recursos, no sólo favorecen al alumnado sordo sino también al oyente. No se puede olvidar que diversificar las fuentes de adquisición del conocimiento contribuye a un mayor desarrollo personal, en cuanto que permite adquirir competencias específicas que proporciona cada modalidad de recurso. Todos los estudiantes, oyentes o no, necesitan potenciar, por ejemplo, la memoria visual, de ahí la importancia de favorecer el uso de la imagen dentro del proceso de enseñanza-aprendizaje. En muchas ocasiones, en nuestras aulas, el monopolio de la palabra, de la oralidad, no facilita la introducción de otros códigos, por ejemplo visuales, o kinestésicos, lo que acaba contribuyendo a que los estudiantes no adquieran conocimientos y competencias que le serían muy útiles en su vida ordinaria y académica. El alumnado con diversidad funcional auditiva necesita trabajar con materiales que le faciliten el acceso al conocimiento utilizando todos los sentidos, incluso, el auditivo dependiendo de las características del estudiante. Téngase en cuenta que este alumnado, en muchas ocasiones, no necesita materiales muy diferenciados al resto de sus compañeros y compañeras, si se opta por metodologías donde se combinen el uso de las dos lenguas, oral y de signos; además de posible uso de recursos ma- 
Una taxonomía de materiales didácticos para la inclusión de alumnado con diversidad funcional auditiva

Carla Vázquez Formoso, María Montserrat Castro Rodríguez y Jesús Rodríguez Rodríguez

nipulativos/táctiles, visuales o informáticos, que faciliten la traducción del lenguaje oral al escrito.

\section{El estudio empírico}

El desarrollo de la parte empírica del trabajo se centró inicialmente en la localización de las diferentes clasificaciones y taxonomías que existen sobre los materiales didácticos y en particular en relación con los materiales relacionados con la sordera. Una vez detectada la no existencia de clasificaciones específicas sobre el tema se procedió a realizar una exhaustiva búsqueda de información sobre propuestas de materiales diseñados y concebidos para atender alumnado con sordera con el propósito de analizarlos y clasificarlos. Veamos a continuación de un modo detallado las fases seguidas en el transcurso de esta parte empírica.

\section{Fase 1. Revisión bibliográfica sobre la discapacidad auditiva y materiales didácticos}

Se procedió inicialmente a una revisión exhaustiva de las revisiones realizadas en la literatura con el propósito de clarificar y sistematizar las diferentes definiciones, clasificaciones e investigaciones relacionadas con el tema de los materiales didácticos y la discapacidad auditiva, prestando especial atención a aquellas que pudiesen estar concebidas para clasificar los materiales didácticos elaborados sobre la discapacidad, o concretamente en la sordera. En la totalidad de los casos las palabras clave utilizadas para realizar las búsquedas fueron las de: sordos, personas sordas, educación de sordos, aprendizaje de sordos, discapacidad auditiva, niños sordos, educación y lenguaje, materiales didácticos, recursos discapacidad auditiva, recursos para sordos, materiales educativos sordos, libros de texto e inclusión educativa.

De un modo concreto se realizaron búsquedas presenciales y virtuales en los siguientes contextos, buscadores y páginas web:

- Bibliotecas de la Universidad de Santiago de Compostela y de la Coruña.

- Bases de datos ISOC y el catálogo Dialnet.

- Bases de datos Scopus y Web of Science. 
- Revistas especializadas en el ámbito de la Tecnología Educativa y de Educación Especial.

- Buscador Google y Google Academic.

- IARTEM (International Association on Textbooks and Educational Media).(www.iartem.org). Mediante la revisión de sus trabajos tuvimos la oportunidad de consultar las publicaciones y congresos realizados en sus 25 años de historia y en los que apenas se recogen propuestas de materiales relacionadas con la sordera.

- CNLSE (Centro de Normalización Lingüística de la Lengua de Signos Española) (www.cnls.es). En su Biblioteca Virtual encontramos alguna guía, tanto para profesionales como para familias, sobre las personas con discapacidad auditiva.

\section{Fase 2. Revisión y clasificación de los materiales y recursos didácticos}

Partiendo de la revisión teórica anterior, en líneas generales detectamos que apenas se recogen clasificaciones realizadas sobre los materiales dirigidos al ámbito de la diversidad funcional y de un modo muy especial en relación con la sordera, por lo que podemos señalar que, en cuanto a los materiales y recursos encontrados, que se encuentran centrados en el desarrollo de las personas con discapacidad auditiva y el entorno que los rodea, trabajamos con un conjunto considerablemente extenso que no cuenta con una clasificación o marco en el que situarlos para poder trabajar con ellos de manera accesible. Por ello, los materiales fueron analizados siguiendo las guías clasificatorias diseñadas específicamente por nosotros para este tipo de recursos. Igualmente, y partiendo de autores que ya trabajaron sobre distintas clasificaciones de los materiales (Area, 2004; Parcerisa, 1996; Principe, 2012), tanto en general como los dirigidos al alumnado con NEAE, se elaboró un borrador de las fichas sobre la con la que clasificaron los materiales de acuerdo con sus características..

En un primer momento, se procedió a contactar con el Centro de Normalización Lingüística de la Lengua de Signos Española (CNLSE), puesto que la biblioteca de recursos con la que cuentan sería el eje central del cual obtener los materiales a analizar, ya que muchas de las asociaciones y colegios españoles utilizan los materiales que allí se encuentran; permitiéndonos acceder a diversos ejemplares de materiales de manera 
Una taxonomía de materiales didácticos para la inclusión de alumnado con diversidad funcional auditiva

Carla Vázquez Formoso, María Montserrat Castro Rodríguez y Jesús Rodríguez Rodríguez

online o presencial, contando con la ayuda de su servicio de asesoramiento documental.

También se consultaron otras fuentes como bancos de recursos en línea o las páginas web de diversas administraciones:

\section{Bancos de recursos en línea}

La busca se inició por el repositorio en línea de la CNLSE, ya que en él se cuenta con recursos web que no pueden ser analizados de manera tangible en papel. Conectado con este repositorio se encuentra el proyecto Mi hijo sordo, de la CNSE en colaboración con la Fundación Mapfre, que nos ofrece diversos recursos en DVD y en línea con el fin de aportarles a las familias diversas guías y ayudas para entender a sus hijos/as sordos a lo largo de su vida; y FIAPAS (Confederación Española de Familias de Personas Sordas), que pretende dar respuesta las necesidades que puedan tener las personas sordas en su día a día; así como las distintas federaciones de personas sordas de cada comunidad autónoma y las asociaciones de padres y amigos de los sordos de las distintas comunidades. En este sentido, resultaron de gran interés las aplicaciones de móvil diseñadas como diccionarios de lengua de signos como el DILSE (Diccionario de Lengua de Signos Española). En concreto en Galicia se accedió también a la página web de la FAXPG (Federación de Asociaciones de Personas Sordas de Galicia), resultando muy útil el Diccionario de Toposignos de Galicia, que recoge los signos de muchos pueblos gallegos.

Conviene tener presente que en el territorio español contamos con dos lenguas de signos reconocidas: la lengua de signos española (LSE) y la lengua de signos catalana (LSC), por lo que muchas personas con discapacidad auditiva de esa región, utilizarán para comunicarse y, por ende, participar en el proceso de enseñanza - aprendizaje, la lengua de signos catalana. Tanto es así, que desde la Generalitat de Catalunya y desde otros organismos como FESOCA (Federación de Persones Sordes de Catalunya), e incluso desde empresas del sector editorial con base en esa comunidad, se producen materiales educativos y apoyos para el aprendizaje de la LSC para este colectivo. Por ejemplo, Carambuco Ediciones, además de publicar cuentos adaptados a la LSE también lo hace en su versión en LSC. Por otro lado, esta comunidad cuenta con el CREDA, que es el servicio de apoyo a las escuelas para adaptar la respuesta 
educativa a las necesidades personales, sociales y curriculares del alumnado con déficit auditivo, trastornos del lenguaje y/o la comunicación; y por ello son los encargados de publicar material educativo en lengua de signos, como pueden ser las láminas de Vocabulario Básico en LSC. Pero, en esta investigación, nos centraremos en la LSE, puesto que es la de uso mayoritario en España.

\section{Administraciones}

Se accedió a las referencias web del Ministerio de Educación, Cultura y Deporte, que cuenta con bancos de recursos como Redined (Red de Información Educativa), Educalab e INTEF (Instituto Nacional de Tecnologías y de Formación del Profesorado), que así mismo incluyen el CEDEC (Centro Nacional de Desarrollo Curricular de Sistemas No Propietarios) y Procomún (Red de Recursos Educativos Abiertos).

Igualmente en el ámbito autonómico, se accedió a las bibliotecas de medios y publicaciones de las diversas Consejerías de Educación de las comunidades autónomas españolas, así como a sus centros de formación del profesorado. En algunos casos, desde las consejerías estaban conectados con los Equipos de Orientación Específicos de las comunidades, Ilegando a tener materiales de elaboración propia en el área de discapacidad auditiva, como son los casos de CREENA (Centro de Recursos de Educación Especial de Navarra), o el Blog León Oye promovido por el Equipo de Orientación Educativa Específico para la Discapacidad Auditiva y el Equipo Específico de Discapacidad Auditiva de Madrid. Conviene destacar igualmente la Biblioteca Signos incluida en el portal Biblioteca Virtual Miguel de Cervantes. En el caso gallego, señalar, por un lado, el Equipo de Orientación Específico especializado en Discapacidades Sensoriales, que nos muestra una guía sobre las mismas; mientras que, por otra banda, en el Repositorio de Contenidos Educativos ABALAR encontramos algunos materiales incluidos bajo la denominación de recursos para la "discapacidad auditiva", "hipoacusia" "diversidad funcional auditiva", aunque su número es reducido y suelen estar relacionados con la simbología del lenguaje de signos. 


\section{Fase 3. Criterios y propuestas de clasificación.}

Esta fase estuvo centrada en el análisis y clasificación de los materiales encontrados. Para la elaboración de la propuesta clasificatoria se partió de los siguientes principios:

- Consideración de propuestas de clasificación mencionadas anteriormente referidas a los materiales didácticos en general y propuestas de clasificaciones dirigidas al alumnado con diversidad funcional.

- La muestra de materiales encontrados, lo que nos permitió añadir nuevas dimensiones a nuestra propuesta.

Conviene destacar que, durante este proceso de búsqueda y análisis de materiales y recursos, se fueron cubriendo las fichas, que se pueden ver en la Tabla 1, diseñadas para este proceso en concreto, con los datos y características de cada uno de los materiales analizados; puesto que, como se mencionó anteriormente, no contábamos con ninguna fuente de este tipo centrada concretamente en los materiales dirigidos a la comunidad sorda. Por ello, la propuesta de clasificación está estructurada en cinco apartados que nos permite recoger los datos básicos sobre los materiales didácticos diseñados para las personas con diversidad auditiva, proporcionándonos, al final, una serie de características que serán las que determinen la taxonomía que se persigue en este estudio.

Tabla 1. Ficha clasificatoria de los materiales didácticos (Elaboración propia). Título del material Datos de identificación

\begin{tabular}{ll}
\hline Autor & \\
\hline Lugar y fecha de publicación & \\
\hline Editorial & \\
\hline Formato & $\frac{\text { Manipulativo }}{\text { Impreso }}$ \\
$\frac{\text { Auditivo }}{\text { Audiovisual }}$ \\
\hline Dixital y/o virtual \\
\hline Resumen & \\
\hline
\end{tabular}




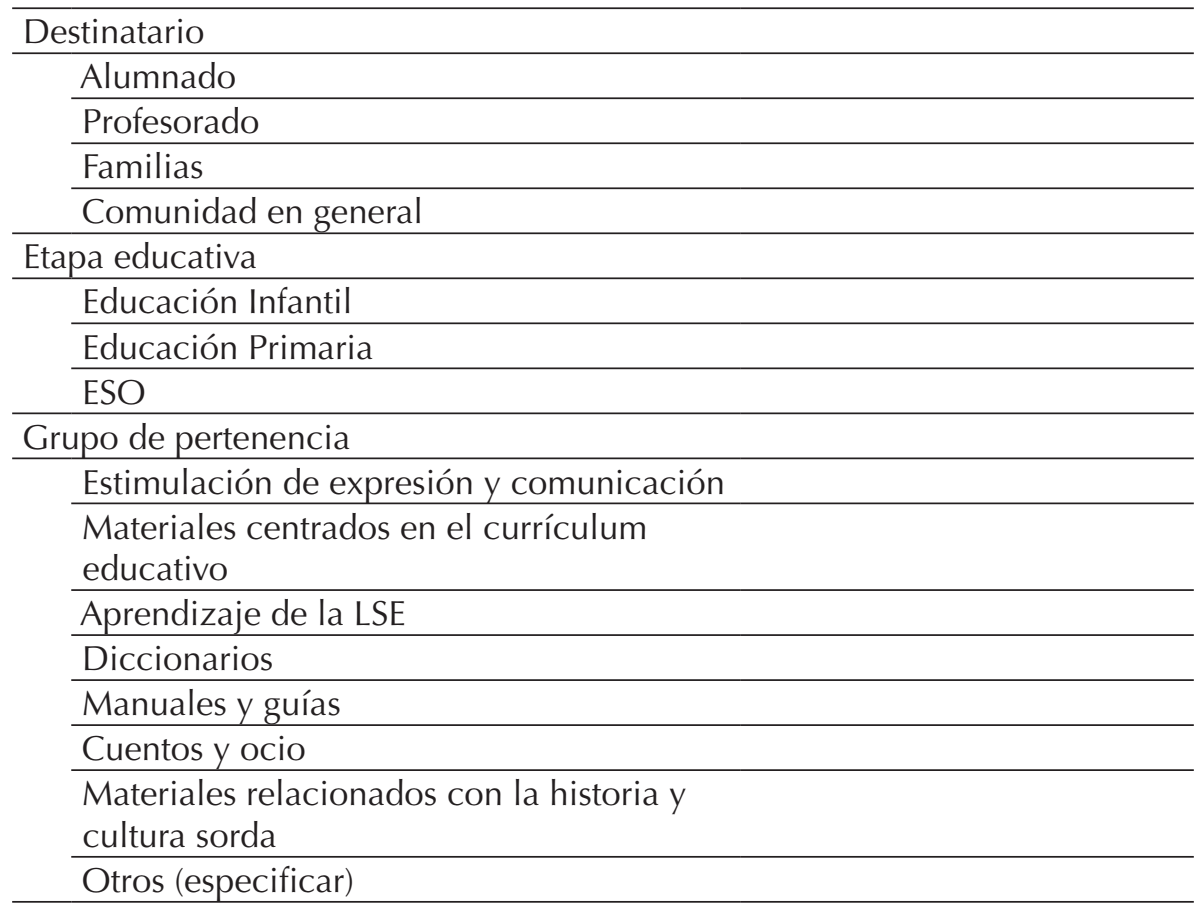

En ellas se señalan las diversas particularidades de cada uno de los materiales en torno a ámbitos como los datos de identificación, destinatarios, formato, etapa educativa o el grupo temático al que pertenece, como puede ser la expresión y la comunicación, el aprendizaje de la LSE, cuentos, diccionarios, materiales centrados en el currículum, diccionarios y manuales, o los centrados en la cultura sorda. Pero, hay que aclarar que durante la fase de agrupamiento de cada uno de los materiales, la propuesta clasificatoria sufrió algunas modificaciones para ir adaptándose a las características de los recursos que estábamos analizando.

La propuesta clasificatoria está pensada y dirigida para todas aquellas personas que precisen hacer uso de materiales didácticos en relación a las personas con discapacidad auditiva y aquellos otros profesionales que necesiten de estos recursos y materiales para hacer uso de ellos con todo el alumnado en un aula concreta. La clasificación está pensada para profesionales de la educación así como para padres/madres, personal de asociaciones, entre otros.

La taxonomía se presenta dando lugar a un conjunto final, pero distribuida en distintos subapartados, como se puede apreciar en la Tabla 2 , según el destinatario principal para el que se presenten los distintos 
recursos. Indicar que los materiales que se recogen en la taxonomía, podrían formar parte de dos o tres grupos generales, ya que, por ejemplo, algunos de los diccionarios podrían ser utilizados por el subgrupo del alumnado de los distintos niveles, así como por la comunidad en general o las familias.

Tabla 2. Clasificación de los materiales didácticos orientada a la discapacidad auditiva (Elaboración propia).

\begin{tabular}{l}
\hline Destinatario \\
\hline Impresos \\
- Cuentos y ocio \\
- Cultura sorda \\
- Expresión y comunicación \\
- Manuales y guías \\
Manipulativos \\
- Cuentos y ocio \\
Audiovisuales \\
- Cuentos y ocio \\
- Currículum educativo \\
- Manuales y guías \\
Digitales \\
- Manuales y guías \\
Impresos \\
- Aprendizaje de la LSE \\
- Cultura sorda \\
- Currículum educativo \\
- Diccionarios \\
- Expresión y comunicación \\
- Manuales y guías \\
Audiovisuales \\
- Aprendizaje de la LSE \\
- Currículum educativo \\
- Expresión y comunicación \\
Digitales y APP \\
- Aprendizaje de la LSE \\
- Currículum educativo \\
- Diccionarios \\
- Manuales y guías \\
\hline Profesorado
\end{tabular}


Alumnado

$$
\begin{aligned}
& \text { Impresos } \\
& \text { - Aprendizaje de la LSE } \\
& \text { - Cuentos y ocio } \\
& \text { - Currículum educativo } \\
& \text { - Diccionarios } \\
& \text { - Expresión y comunicación } \\
& \text { - Manuales y guías } \\
& \text { Manipulativos } \\
& \text { - Cuentos y ocio } \\
& \text { - Currículum educativo } \\
& \text { Audiovisuales } \\
& \text { - Aprendizaje de la LSE } \\
& \text { - Cuentos y ocio } \\
& \text { - Currículum educativo } \\
& \text { - Otros } \\
& \text { Digitales y APP } \\
& \text { - Aprendizaje de la LSE } \\
& \text { - Cultura sorda } \\
& \text { - Currículum educativo } \\
& \text { - Diccionarios }
\end{aligned}
$$

Otros

\begin{tabular}{cc}
\hline Impresos \\
- Aprendizaje de la LSE \\
- Cuentos y ocio \\
- Cultura sorda \\
- Diccionarios \\
- Manuales y guías \\
Audiovisuales \\
- Aprendizaje de la LSE \\
- Cuentos y ocio \\
Digitales y APP \\
- Aprendizaje de la LSE \\
- Cuentos y ocio \\
- Diccionarios \\
- Manuales y guías \\
Otros \\
\hline
\end{tabular}

Teniendo en cuenta el destinatario al que va dirigido principalmente el material y/o recurso, otro aspecto a tener en cuenta son las subcategorías que nos permitirán especificar más allá del destinatario el ámbito de uso preferente para cada uno. Por ello, y partiendo de los materiales trabajados y analizados, así como de la ficha clasificatoria mencionada anteriormente resultante del análisis de investigaciones anteriores en el 
Una taxonomía de materiales didácticos para la inclusión de alumnado con diversidad funcional auditiva

Carla Vázquez Formoso, María Montserrat Castro Rodríguez y Jesús Rodríguez Rodríguez

campo de los materiales, se establece la siguiente tipología de materiales:

- Materiales para la estimulación de la expresión y la comunicación.

- Materiales centrados en el currículum educativo.

- Materiales para el aprendizaje de la Lengua de Signos.

- Diccionarios.

- Manuales y guías.

- Cuentos y ocio.

- Materiales relacionados con la historia y cultura sorda.

Cabe destacar que los recursos que aparecen recogidos son diseñados y publicados a partir del año 2000 y presentan como destinatarios fundamentales el ámbito educativo, siendo fundamentalmente guías, manuales, diccionarios y propuestas curriculares. De la misma manera, algunos de ellos se publicaron con formato impreso ya que ese es su formato original en el que fueron publicados y en la red se pueden encontrar en formato PDF o escaneados.

La gran mayoría de los materiales clasificados y analizados se corresponden con la etapa educativa de Primaria. Conviene destacar que es a partir del año 2000 cuando está en pleno proceso de implantación la LOGSE, que tiene entre sus principios la atención al alumnado con NEAE y es cuando se produce una incorporación del alumnado con diversidad funcional auditiva a los centros ordinarios, lo que generó nuevas necesidades dentro del aula ordinaria. Tampoco se puede olvidar que paulatinamente, entre una parte de la sociedad, se generó una mayor sensibilización para mejorar los procesos de inclusión educativa. La incorporación de las TIC en los centros educativos ordinarios abrió nuevas posibilidades de usar diferentes materiales y recursos, al mismo tiempo que favorece incorporar antiguos e interesantes recursos en nuevos soportes puesto que permitió proporcionar un aprendizaje en igual medida para todo el alumnado en el aula, evitando barreras de comunicación que, en el caso del alumnado con discapacidad auditiva están centradas, fundamentalmente, en el acceso al aprendizaje comunicado de manera oral en el aula. Incorporar las tecnologías en el aula, utilizando el software adecuado permitirá que el contenido sea accesible para este colectivo, puesto que la información puede "perderse" en situaciones como al escribir de espaldas al alumnado en el encerado, ya que deja al alumnado sordo sin la posibilidad de la lectura labial 
como único código de acceso al conocimiento. En este sentido se están desarrollando proyectos como los libros de texto híbridos en Grecia o el APEINTA en España.

\section{La clasificación de los materiales}

Veamos a continuación una síntesis de los materiales encontrados y una valoración de los mismos tomando como eje central para ello los destinatarios a los que están dirigidos cada grupo de materiales y recursos, permitiendo de esta manera que su localización y posterior uso se realice de una manera más accesible.

Esta decisión de establecer los materiales en torno a su destinatario principal, que no el único, está justificada debido a que, a la hora de buscar materiales para trabajar con el alumnado con discapacidad auditiva, tanto dentro como fuera del aula, permitiría a los distintos usuarios acceder directamente al grupo de materiales y recursos centrados o dirigidos a su campo de trabajo directo, acotando así la búsqueda y permitiendo que toda la comunidad pueda acceder de manera más rápida y sencilla a estos materiales.

A continuación se presenta, por tanto, la taxonomía distribuida ${ }^{2}$ en función de los distintos destinatarios señalados: familias, profesorado, alumnado y comunidad en general; mostrando en cada uno de ellos las principales tipologías de materiales y recursos encontrados para su trabajo en cada ámbito.

\section{Materiales dirigidos a familias}

En este caso, en la revisión realizada se han encontrado materiales impresos, manipulativos y audiovisuales. Veamos a continuación las subcategorías definitivas en este apartado y la tipología de recursos encontrados:

Cuentos y ocio: engloba en su mayoría cuentos impresos, como la colección de Carambuco cuentos, aunque también películas como Los

2 Para una consulta detallada del conjunto de los materiales y su clasificación hemos elaborado la siguiente página web en la que pueden consultar los recursos estudiados: https://taxmaterialsordos.wordpress.com/ 
Una taxonomía de materiales didácticos para la inclusión de alumnado con diversidad funcional auditiva

Carla Vázquez Formoso, María Montserrat Castro Rodríguez y Jesús Rodríguez Rodríguez

ladrones de motores y hasta puzles y juegos con la Colección de Animacuentos para las familias con niños menores de cinco años.

Manuales y guías: encontrados de manera impresa, audiovisual o digital, presentan diversas propuestas y consejos para ayudar a este colectivo. En cuanto al formato digital está representado en su totalidad por la página web Mi hijo sordo, cuyo contenido aporta a las familias información y orientación sobre la experiencia de contar con un miembro sordo en la unidad familiar.

Por otro lado, las guías impresas se centran en el mismo aspecto pero siendo redactadas por diversos colectivos y, en algunas ocasiones, dando respuestas más concretas a preguntas como ¿Qué harías si un miembro de tu familia fuera sordo?.

Los tres ámbitos restantes, en cuanto a los grupos de pertinencia señalados anteriormente y en relación con las familias, currículum educativo, expresión y comunicación y cultura sorda, están en su totalidad publicados de manera impresa y audiovisual. En este sentido puede sorprender que, en el ámbito familiar, veamos materiales centrados en el currículum educativo, pero esto es debido a que materiales como Signando en primaria: recursos para el cole y la casa nos proporcionan precisamente lo que su título indica, materiales que pueden ser utilizados tanto dentro como fuera del aula.

En cuanto a la expresión y comunicación y el conocimiento de la cultura sorda, se trata de materiales que pueden aportarles a las familias información sobre un miembro sordo de las mismas, así como ayudarlos en el ámbito de la comunicación entre ellos, como hace el recurso impreso Preguntas y respuestas sobre el implante coclear.

\section{Materiales dirigidos para el profesorado}

Los recursos publicados dirigidos al profesorado pueden encontrarse, al igual que anteriormente, de manera impresa, audiovisual y digital, e incluyendo en este caso aplicaciones móviles de las que también se podría hacer uso en el aula. En este caso, los más interesantes son los centrados en el currículum educativo y los manuales y guías, puesto que conforman el grupo más numeroso del que podrán hacer uso profesores/as en su día a día en el centro educativo.

De esta manera, distinguimos materiales pensados para las etapas 
educativas desde Educación Infantil hasta Educación Secundaria Obligatoria; pero el período de Educación Primaria es el que cuenta con más variedad de recursos y materiales. Señalamos a continuación las subcategorías correspondientes:

Curriculum educativo: en relación a los recursos para los docentes que cuenten en su aula con alumnado con discapacidad auditiva, señalamos los que encuentran en formato digital, así como impresos y audiovisuales. En formato digital están englobados dentro de diferentes Equipos de Orientación Específicos que adaptan y publican recursos en relación a materias como conocimiento del medio o lengua para Educación Primaria, permitiendo así que cualquier docente pueda acceder a ellos a través de internet.

Los impresos y audiovisuales, de igual manera, le ofrecen al cuerpo docente diversos recursos que pueden considerar a la hora de contar con un alumno sordo en su aula, como son las publicaciones del gobierno Vasco sobre el Acceso del alumnado con sordera al currículum de lenguas.

Manuales y guías: publicados de manera impresa suelen proporcionarle al profesorado, de manera general, una orientación o pautas para actuar en el momento en que el alumnado sordo se incorpora a su aula. Señalamos, por ejemplo, la publicación Discapacidad auditiva: orientación para la intervención educativa, que le aportará a los docentes consejos sobre cómo comenzar a trabajar con el alumnado con discapacidad auditiva.

Expresión y comunicación y cultura sorda: los recursos encontrados que potencian la expresión y comunicación le permiten al profesorado conocer técnicas para eliminar las barreras de comunicación en el aula con el alumnado sordo, como Entre tú y yo ya no hay barreras. En relación con la cultura sorda, está reflejada en el documento Sensibilización en contextos educativos, que le permite al cuerpo docente, y al resto del alumnado, comprender al alumnado sordo que estudie en el centro.

Aprendizaje de la lengua de signos: los materiales impresos y audiovisuales que se corresponden con el aprendizaje de la lengua de LSE, están ligados a colecciones como Vamos a signar en diferentes niveles de la lengua, constando el recurso de un libro impreso y un material audiovisual (DVD) por el que seguir cada una de las lecciones que en ellos se recogen. Estos materiales pueden, por tanto, contribuir con metodologías y actividades que el profesorado puede poner en práctica en 
el aula para que todo el alumnado, sordo y oyente, pueda aprender la LSE. Y, digitalmente, Cervantes Virtual, se convierte en una importante fuente de recursos para el aprendizaje de esta lengua.

Diccionarios: publicados para distintas edades y niveles de aprendizaje de la LSE, por lo que se encuentran muy ligados a la categoría anterior, se presentan como un material complementario con una fuerte presencia en el aula, ofreciendo una gran versatilidad a la hora de trabajar con ellos ya que se encuentran en formato impreso, digital o en aplicaciones móviles. En concreto destacamos Mis primeros signos. Diccionario de LSE para niños y niñas o el Diccionario Normativo de la Lengua de Signos Española.

\section{Materiales dirigidos al alumnado}

Estrechamente ligados con la categoría de profesorado, se encuentran los recursos destinados al alumnado, con los que pueden trabajar de manera autónoma o en el aula con el resto de sus compañeros. Entre las subcategorías, en este caso, encontramos:

Cuentos y ocio: el ocio, configurando una parte fundamental del desarrollo de cualquier niño o niña, cuenta, en el ámbito de la discapacidad auditiva con diferentes recursos para utilizar. Se encuentran en tres formatos: impreso, audiovisual y manipulativo, correspondiendo a este último formato la colección, ya antes mencionada en para su uso familiar, Animacuentos, que intenta fomentar la pasión por la lectura en todos los niños y niñas sordos. Y, por otro lado, los cuentos, pueden ser tradicionales adaptados a la lengua de signos, o, especialmente producidos para este colectivo con su versión en LSE.

Manuales y guías y cultura sorda: a pesar de que la mayoría de materiales están producidos para Educación Primaria, cabe destacar que también se produjo material de este tipo para el alumnado adolescente. Ambos tratan temas que pueden ser relevantes tanto para la juventud sorda como oyente, pero estando adaptados a los primeros, como puede ser el material digital Mi blog paso a paso en LSE, o las publicaciones relativas a los cuidados en internet o la prevención de drogas.

Currículum educativo: los materiales y recursos que conforman esta subcategoría se encuentran producidos de manera impresa, audiovisual, manipulativo o digital, sobresaliendo este último puesto que se llegaron 
a producir aplicaciones móviles como AUDAGE, una APP que le permite al alumnado sordo comprender el ciclo del agua en educación primaria.

Consideramos, en este sentido, que estos materiales pueden ser trabajados en el aula como parte de actividades propuestas por el profesorado, o, por el contrario, podrían ser también utilizadas de manera autónoma fuera del horario escolar. Por ejemplo, Contamos con signos, material impreso producido por la FAXPG, presenta cuentos, actividades, libros, etc.; para ayudar al alumnado sordo en su aprendizaje de la lectura.

Diccionarios y aprendizaje de la lengua de signos: al igual que en ocasiones anteriores, los materiales de ambas subcategorías, se encuentran en formato mayoritariamente impreso, aunque también lo hacen de manera audiovisual y digital. Señalamos la página web Actividades en LSE que permite aprender, en línea y de manera autónoma, la LSE, y apoyándose en diccionarios, sobre todo en línea y aplicaciones móviles que permiten visualizar vídeos en los que se muestra cómo se signan las palabras, pueden construir una herramienta de aprendizaje muy sólida.

\section{Materiales dirigidos a la comunidad en general}

Esta categoría final, dedicada, coloquialmente, a la comunidad en general, está formada por materiales y recursos que pueden ser utilizados por cualquier persona que esté interesada en acercarse a la comunidad sorda, al aprendizaje de la lengua de signos o que conviva en su entorno con personas con discapacidad auditiva.

Cultura sorda: en este sentido, se encuentran en el mercado diversos materiales impresos dedicados a dar a conocer más en profundidad a este colectivo, como puede ser el boletín Protagonistasordo de la FAXPG.

Aprendizaje de la lengua de signos: el aprendizaje de esta lengua, al igual que otras, se encuentra abierta a toda la comunidad, por lo que encontramos materiales digitales, audiovisuales o impresos que pueden servir de apoyo para esta tarea. Las federaciones de personas sordas publican carteles en los que se presentan, por ejemplo, los alfabetos dactilológicos, y, en la red, encontramos varios recursos a los que se puede acceder para aprender esta lengua. Así mismo, recursos como el Diccionario de Topónimos Galego, le proporciona a la comunidad una manera simpática y atractiva como reclamo para comenzar el aprendizaje de la lengua de signos. 
En cuanto al resto de subcategorías, estimulación y expresión de la comunicación, materiales centrados en el currículum, diccionarios, manuales y guías o cuentos y ocio, vemos cómo se tienen producido diversos materiales generales para quien necesite hacer uso de los mismos, como manuales y guías o diccionarios y material complementario, que le aportan a esta comunidad y al público en general diversos conocimientos adaptados en áreas muy variadas, como manuales de conducción, guías turísticas o la prevención de drogas.

\section{Conclusiones}

A modo de síntesis, presentamos algunas de las principales conclusiones que se derivan de la investigación realizada y de la taxonomía propuesta:

- Creemos que a través del trabajo realizado se ha evidenciado de un modo claro y contundente, las necesidades y carencias de recursos existentes sobre esta temática.

- Se trata de uno de los primeros trabajos que ha permitido centralizar una buena parte de los bancos de recursos existentes hasta el momento sobre la temática y la sordera, lo que puede resultar de gran ayuda al profesorado, familias y otros profesionales.

- La propuesta planteada ha ayudado a completar otro tipo de clasificaciones existentes sobre los materiales y que en la mayoría de los casos no recogían las características y las particularidades de los materiales existentes para niños sordos. La taxonomía ha detectado los ámbitos en los que hay más necesidades o carencias de recursos como es el caso de materiales específicos para etapas educativas no obligatorias; sin olvidar que la mayoría de ellos, realizados en España, están elaborados en español, existiendo carencias importantes en el resto de las lenguas peninsulares, excepto el catalán.

- La existencia de la taxonomía elaborada, abre posibilidades futuras para el estudio e investigación de los materiales didácticos existentes para trabajar el tema de la sordera. La falta de una clasificación impedía que pudiese realizarse un estudio sistematizado sobre los recursos existentes en este ámbito, algo que resulta imprescindible analizar debido al impacto que tienen en el pro- 
ceso de enseñanza-aprendizaje. En muchos casos se desconoce si introducen contenidos adecuados, si comparten estereotipos y estigmas sociales, sean con relación a estereotipos de género, culturales, etc. como ocurre con otros materiales elaborados para todo tipo de estudiantes.

- Conviene destacar que el trabajo ha permitido detectar carencias de materiales y recursos existentes sobre el tema y facilita que la propuesta puede constituirse en un referente importante para editoriales y productores de materiales didácticos. Se echa en falta una diversificación de los productores de materiales, quedando restringidos, a colectivos específicamente relacionados con este tipo de diversidad. Esta situación pone en evidencia la todavía deficitaria inclusión del alumnado con ciertas necesidades educativas en proyectos editoriales o de otras instituciones, lo que puede restringir las posibilidades de incorporar temáticas que puedan ser de interés general. Igualmente, el trabajo ha permitido identificar las funciones que pueden cumplir los materiales didácticos existentes sobre la sordera y que pueden resultar de ayuda en el momento de tener que seleccionar propuestas de trabajo sobre el tema.

- Visibilización de materiales producidos por los propios profesores y que en una buena parte de los casos son desconocidos. El estudio realizado ha permitido poner de relevancia el papel de las TIC en el diseño y desarrollo de propuesta de materiales didácticos para alumnado con sordera. El estudio detallado ha puesto de manifiesto las grandes posibilidades que introduce la tecnología para adaptar los materiales a las nuevas necesidades del alumnado con discapacidad auditiva que pretende incorporarse plenamente a la sociedad en igualdad de derechos, al mismo tiempo que puede facilitar la superación de la brecha que tiene este colectivo para acceder a ciertas temáticas, sobre todo, en una sociedad en la que la palabra oral sigue teniendo un gran prestigio y poder.

- Igualmente, el trabajo ha permitido poner de relieve la necesidad de favorecer e impulsar estrategias que ayuden en los procesos de adaptación de los materiales "convencionales" a las particulares de los niños sordos. Al mismo tiempo permite superar ciertos posicionamientos estereotipados o clasistas, que restringían el uso de recursos elaborados específicamente para el alumnado con dis- 
Una taxonomía de materiales didácticos para la inclusión de alumnado con diversidad funcional auditiva

Carla Vázquez Formoso, María Montserrat Castro Rodríguez y Jesús Rodríguez Rodríguez

capacidad auditiva, y pudiendo constatarse que muchos de ellos serían muy útiles para el resto del alumnado, debido a que se trabajan temáticas, objetivos y competencias apropiadas para todos los estudiantes, lo que facilita mucho su uso en el aula ordinaria, a la que asisten estudiantes con o sin necesidades específicas en el ámbito auditivo. Téngase en cuenta, que una de las características fundamentales de la mayoría de materiales hacen uso de técnicas gráficas y visuales muy válidas para todo el alumnado, que facilitan el acceso al conocimiento desde perspectivas de aprendizaje diferentes.

Para finalizar, decir que el estudio realizado ha servido para evidenciar de un modo claro las limitaciones que presentan los libros de texto, los cuales de un modo nulo o muy precario contemplan las particularidades del niño sordo. Creemos que la clasificación obtenida, conforma, una valiosa fuente de información para todos aquellos profesionales, familias y/o personas sordas que quieren trabajar distintos aspectos en materia de discapacidad auditiva.

\section{Referencias bibliográficas}

ÁFIO A., CARVALHO AT, CARVALHO LV, SILVA ASR, PAGLIUCA LMF (2016). Accessibility assessment of assistive technology for the hearing impaired. Revista Bras Enferm; 69 (5):781-7. DOI: http://dx.doi.org/10.1590/0034-7167.2016690503

AREA MOREIRA, M. (2004). Los medios y las tecnologías en la educación. Madrid: Pirámide.

AREA MOREIRA, M. (2009). Introducción a la tecnología. La Laguna: Universidad de la Laguna. Creative Commons.

BARRAGÁN, C. (sf). Recursos materiales para alumnado con necesidades educativas especiales ligadas a un déficit motor. Vitoria: Departamento de Educación, Universidades e Investigación del Gobierno Vasco.

BOOTH, T. y AINSCOW, M. (2002). Guía para la evaluación y mejora de la educación inclusiva. Desarrollando el aprendizaje y la participación en las escuelas. CSIE y Consorcio Universitario para la Educación Inclusiva.

CABERO, J. (2001), Tecnología Educativa, Diseño y Utilización de Medios para la Enseñanza. Madrid: Paidós.

DE MARTINO, J.M. y otros (2016). Singning avatars: making education more inclusive. Universal Access in the Information Society. 16, 1-16. DOI: 10.1007/s10209-0160504-x

DELGADO DE PAIVA, M. (2009). As dificultade de aprendizage e os materiais curricula- 
Carla Vázquez Formoso, María Montserrat Castro Rodríguez y Jesús Rodríguez Rodríguez

res. Um estudo dos manuais escolares do primeiro ciclo do ensino básico. Departamento de Didáctica y Organización Escolar. Universidad de Santiago de Compostela. Tesis Doctoral.

DOMINGO SEGOVIA, J. y PEÑAFIEL MARTINEZ, F. (1998). Desarrollo curricular y organización en la escolarización del niño sordo. Málaga: Aljibe.

GARRIDO, C., MARTÍNEZ, I. y VÁZQUEZ, A. (1990). La integración de los niños sordos en el sistema escolar. Infancia y Aprendizaje: Journal for the Study of Education and Development (50), 43 - 62. Recuperado de https://dialnet.unirioja.es/servlet/ articulo?codigo $=48349$

IGLESIAS, A., JIMÉNEZ, J., REVUELTA, P. y MORENO, L. (2014). Avoiding communication barriers in the classroom: the APEINTA project. Interactive Journal Environments, $22,928-843$.

PARCERISA ARAN, A. (1996). Materiales curriculares. Cómo elaborarlos, seleccionarlos y usarlos. Barcelona: Graó.

PRÍNCIPE ALONSO, L. (2012). Análisis de los materiales educativos existentes para las enseñanzas de la LSE como segunda lengua de 0 a 12 años (Trabajo Fin de Máster). Universidad de Valladolid. Valladolid.

RODRÍGUEZ CUELLO, E. (2011). Ayudas técnicas para la comunicación y recursos materiales y personales para la atención de los alumnos/as con necesidades educativas especiales (ACNEE). Didacta 21. Revista Digital: Reflexiones y Experiencias Innovadoras en el Aula, 30, 1-10.

RODRÍGUEZ RODRÍGUEZ, J. (2001). Os materiais curriculares impresos. Santiago de Compostela: Tesis Doctorial, Universidad de Santiago de Compostela.

RODRÍGUEZ RODRÍGUEZ, J. y RODRÍGUEZ REGUEIRA, N. (2016). Revisión de la investigación publicada sobre el libro de texto digital en revistas, publicaciones y congresos internacionales de referencia. Profesorado. Revista de curriculum y formación del profesorado, 20, 1, 9-31. 
